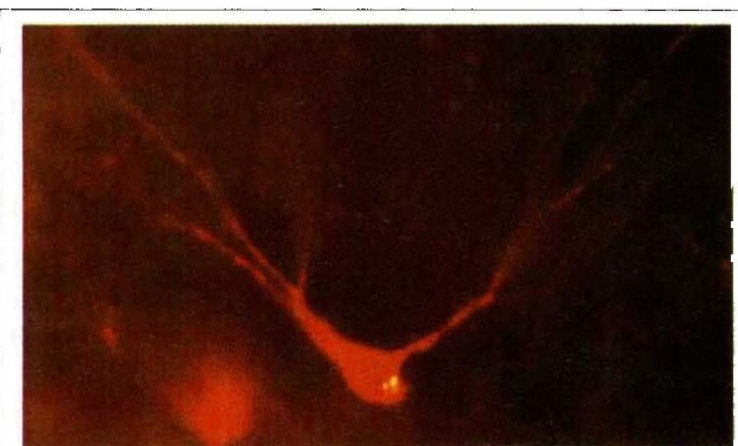

High-power

photomicrographs of individual

hippocampal stem implantation into the dentate gyrus

granule cell-layer of a young rat. The cells have started to

differentiate in ways characteristic of the host tissue, showing arborization into the molecular layer and a single process

extending down into the hilar region.

(Photographs from

ref. 1 courtesy of

Miles Cunningham.) cells 12 days after

from the host. Analysis of heterochronic grafts - that is, grafts into earlier or later host developmental stages might distinguish between these alternatives.

A similar dichotomy applies to the regional specificity of differentiation. In isotopic grafts, the appropriateness of the phenotypes could reflect environmental instruction of a multipotential progenitor or expression of a limited repertoire in a permissive environment. Here, however, Renfranz et al. ${ }^{1}$ have already performed the crucial experiment. They implanted cells of their hippocampal line into the cerebellum, and showed that the engrafted cells survive, proliferate moderately and differentiate. Strikingly, the implanted cells look not at all like hippocampal cells, but acquire unmistakable morphologies of cerebellar neurons (granule and basket cells) and glia (Bergmann glia). The inescapable conclusions are that a single

few cases, being tested) range from Alzheimer's to Parkinson's to epilepsy. Because it is difficult to obtain primary cells in large numbers, and because such cells are notoriously difficult to render transgenic, the search for alternatives has been intense. Here, too, the new cell lines, and others like them, may prove useful.

What do the results of Renfranz et al. and Snyder et al. tell us about the interplay of the intrinsic predilections and intercellular signals that regulate phenotypic choice in the brain? Observations such as these are often taken to support an 'instructive' model: that the engrafted cells are initially plastic, and that their fate is determined by factors present in the host. In fact, however, isotopic and isochronic grafts do not rule out the alternative possibility that the cells are already programmed, and that the host environment is merely permissive. In an instructive model, the hippocampus and cerebellum (which are unusual in bearing large populations of neurons that are generated in early postnatal life) might contain a developmentally regulated series of factors that first stimulate the proliferation of neuroblasts, then cause them to withdraw from mitosis, and finally induce their differentiation. Alternatively, however, this entire sequence may have been programmed into the engrafted cells, requiring only the appropriate trigger

\section{Beam me up}

THE identification of $\mathrm{C}_{60}$ fullerene emerged from a search for interstellar carbon compounds. So it is appropriate that researchers at the Jet Propulsion Laboratory are now looking at the molecule as a fuel for ion-propulsion engines to be used in spacecraft and satellites (S. D. Leifer et al., J.

Propulsion and Power, submitted). Such engines, rather than harnessing the chemical power released in burning rocket fuel, would use the thrust from accelerated ion beams to drive spacecraft or steer satellites. Xenon is one favoured fuel for ion propulsion. But $\mathrm{C}_{60}$ has a mass (720 atomic units) 5.5 times larger, a projected ion-production cost less than half as large ( 90 electronvolts per ion), and an ionization cross-section 17.5 times larger $\left(90 \AA^{2}\right)$. It is also easily stored. Leifer et al. will shortly start experiments to measure the ionization properties of $\mathrm{C}_{60}$ under realistic conditions and investigation of its behaviour in a plasma discharge.

\section{All spruced up}

THE discovery of well-preserved stems of what seem to be conifers from Upper Carboniferous rocks in the West Midlands of England suggests that the origins of conifers must be sought somewhere in the middle of the period. The structures, which are some 300 million years old, were preserved in exquisite detail by a volcanic ashfall They have key conifer characteristics, although they are hard to tell apart from stems of the extinct and possibly related cordaites (J. Galtier et al., Proc. R. Soc. Lond. B247, 211-214; 1992).

Nevertheless, the stems complement an earlier find of fossilized leafy conifer twigs from slightly older rocks, thought to be the earliest-known record of conifers in the fossil record.

\section{On high}

THE amphetamine derivative MDMA familiar to acid-house freaks as ecstasy - may owe both its behavioural and toxic effects to a high selectivity for 5-hydroxytryptamine (5-HT) transporter proteins (G. Rudnick and S. Wall, Proc. natn. Acad. Sci. U.S.A. 89, 1817-1821. 1992). The bizarre tactile enhancement experienced by devotees is not produced by amphetamine itself, which is more selective for catecholamine transporters. MDMA causes release of 5-HT which correlates with the morphological extent of neurotoxic damage. Rudnick and Wall show that MDMA stimulates 5-HT efflux through plasma-membrane and vesicular transporters responsible for its uptake and storage in nerve terminals. Its high potency and stereospecificity correlate with pharmacological data from behavioural studies, suggesting that this interaction may be why the drug is abused. 